\title{
AN IMPROVEMENT AND GENERALIZATION OF BELLMAN- SHAPIRO'S THEOREM ON A PROBLEM IN ADDITIVE NUMBER THEORY
}

S. C. TANG

1. Introduction and two important lemmas. Let $K>1$ be a fixed positive integer. Then we can use any integer $x$ to be uniquely represented as follows:

$$
x=a_{1} K^{n_{1}}+a_{2} K^{n_{2}}+\cdots+a_{t} K^{n_{t}} .
$$

$n_{1}>n_{2}>n_{3}>n_{4}>\cdots>n_{t} \geqq 0$ are integers; so are $a_{1}, a_{2}, a_{3}, \cdots, a_{t}$ each of which is no larger than $K-1$. We set $\alpha(x)=\sum_{i=1}^{t} a_{i}$ and $A(x)=\sum_{y \leq x} \alpha(y)$. In the case of $K=2$, R. Bellman and $\mathrm{H}$. Shapiro have proved the following relation [1]:

$$
A(x)=\frac{x \log x}{2 \log 2}+O(x \log \log x) .
$$

In this paper we extend their result to a general case and discover a better alternative residual.

LEMMA 1.

$$
A\left(K^{n}\right)=\frac{n K^{n}(K-1)}{2}+1 .
$$

ProOF.

$$
\begin{aligned}
A\left(K^{n}\right)= & \sum_{x \leq k^{n}} \alpha(x)=\sum_{x \leq K^{n-1}} \alpha(x)+\sum_{K^{n-1}<x \leq 2 K^{n-1}} \alpha(x)+\cdots \\
& +\sum_{(K-1) K^{n-1}<x<K^{n}} \alpha(x) \\
= & \sum_{x \leq K^{n-1}} \alpha(x)+\sum_{x \leqq K^{n-1}} \alpha\left(K^{n-1}+x\right)+\cdots \\
& +\sum_{x \leqq K^{n-1}} \alpha\left[(K-1) K^{n-1}+x\right] \\
= & \left(A K^{n-1}\right)+K^{n-1}+A\left(K^{n-1}\right)+\cdots+(K-L) K^{n-1} \\
& +A\left(K^{n-1}\right)-(K-1) \\
= & K A\left(K^{n-1}\right)+K^{n-1}+1+\cdots+(K-1)-(K-1) \\
= & K A\left(K^{n-1}\right)+K^{n-1} \frac{K(K-1)}{2}-(K-1) \\
= & K A\left(K^{n-1}\right)+\frac{1}{2}(K-1)\left(K^{n}-2\right) .
\end{aligned}
$$

Received by the editors September 9, 1961. 
We replace $n$ with $n-1, n-2, \cdots, 1$, and multiple $K, K^{2}, \cdots$, $K^{n-1}$ in the order and again add to the above formula. Then we have (to pay attention to $A(K)=A(1)=1$ )

$$
\begin{aligned}
A\left(K^{n}\right) & =K^{n}+\frac{1}{2}(K-1)\left[n K^{n}-2\left(1+K+\cdots+K^{n-1}\right)\right] \\
& =K^{n}+\frac{1}{2}(K-L)\left[n K^{n}-2 \frac{K^{n}-1}{K-1}\right] \\
& =\frac{1}{2} n K^{n}(K-1)+1 .
\end{aligned}
$$

LEMMA 2.

$$
A\left(j \cdot K^{n}\right)=n\left[\frac{j \cdot K^{n}(K-1)}{2}\right]+\frac{j(j-1)}{2} K^{n}+j, \quad j \leqq K-1 .
$$

ProOF.

$$
\begin{aligned}
A\left(j \cdot K^{n}\right)= & \sum_{x \leq j \cdot K^{n}} \alpha(x)=\sum_{x \leq K^{n}} \alpha(x)+\sum_{K^{n}<x \leq 2 \cdot K^{n}} \alpha(x)+\cdots \\
& +\sum_{(j-1) K^{n}<x \leq j \cdot K^{n}} \alpha(x) \\
= & \sum_{x \leq K^{n}} \alpha(x)+\sum_{K^{n}<x \leq 2 K^{n}} \alpha(x)+\cdots \\
& \quad+\sum_{x \leq K^{n}} \alpha\left[(j-1) K^{n}+x\right] \\
= & A\left(K^{n}\right)+K^{n}+A\left(K^{n}\right)+\cdots+(j-1) K^{n}+A\left(K^{n}\right) \\
= & j \cdot A\left(K^{n}\right)+K^{n}(1+\cdots+j-1) .
\end{aligned}
$$

We substitute the result of Lemma 1 into this and simplify in order to get the result we want.

\section{Proof of Theorem 1.}

THEOREM 1. For any positive integer $K \geqq 2$,

$$
A(x)=\frac{K-1}{2} \frac{x \log x}{\log K}+O(x) .
$$

The above residual is the best possible.

Proof. Now we assume: $x=a_{1} K^{n_{1}}+a_{2} K^{n_{2}}+\cdots+a_{t} K^{n_{t}}, n_{1}$ $>n_{2} \cdots>n_{t} \geqq 0$. Then, 


$$
\begin{aligned}
& A(x)=\sum_{y \leq x} \alpha(y)=\sum_{y \leq a_{1} K^{n_{1}}} \alpha(y)
\end{aligned}
$$

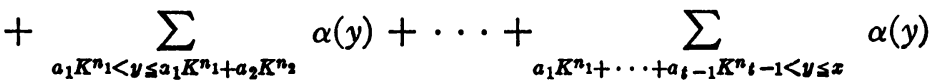

$$
\begin{aligned}
& =A\left(a_{1} K^{n_{1}}\right)+\sum_{y \leq a_{2} K^{n_{2}}} \alpha\left(a_{1} K^{n_{1}}+y\right)+\cdots \\
& +\sum_{\nu \leq a_{t} K^{n_{t}}} \alpha\left(a_{1} K^{n_{1}}+\cdots+a_{t-1} K^{n_{t}-1}+y\right) \\
& =A\left(a_{1} K^{n_{1}}\right)+a_{1} a_{2} K^{n_{2}}+A\left(a_{2} K^{n_{2}}\right)+\cdots \\
& +\left(a_{1}+\cdots+a_{t-1}\right) a_{t} K^{n_{t}}+A\left(a_{t} K^{n t}\right) \\
& =\sum_{i=1}^{t} A\left(a_{i} K^{n_{i}}\right)+\sum_{i=2}^{t}\left(a_{1}+\cdots+a_{i-1}\right) a_{i} K^{n_{i}} \\
& =\sum_{i=1}^{t}\left[n_{i} \frac{a_{i} K^{n_{i}}(K-1)}{2}+K^{n_{i}} \frac{a_{i}\left(a_{i}-1\right)}{2}+a_{i}\right] \\
& +\sum_{i=2}^{t}\left(\sum_{j=1}^{i-1} a_{j}\right) a_{i} K^{n_{i}} \\
& =\frac{n_{1}(K-1)}{2} \sum_{i=1}^{t} a_{i} K^{n_{i}}-\frac{K-1}{2} \sum_{i=1}^{t}\left(n_{1}-n_{i}\right) a_{i} K^{n i} \\
& +\frac{1}{2} \sum_{i=1}^{t} a_{i}\left(a_{i}-1\right) K^{n_{i}}+\sum_{i=1}^{t} a_{i}+\sum_{i=2}^{t}\left(\sum_{j=1}^{i-1} a_{j}\right) a_{i} K^{n_{i}} \\
& =t_{1}-t_{2}+t_{3}+t_{4}+t_{5} \text {. } \\
& t_{1}=\frac{n_{1}(K-1)}{2} x=\frac{(K-1)}{2}\left[\frac{\log x}{\log K}\right] x=\frac{K-1}{2} \frac{x \log x}{\log K}+O(x) \text {; } \\
& t_{2}=\frac{K-1}{2} \sum_{i=1}^{t}\left(n_{1}-n_{i}\right) a_{i} K^{n_{i}} \leqq \frac{(K-1)^{2}}{2} \sum_{i=1}^{t}\left(n_{1}-n_{i}\right) K^{n_{i}} \\
& \leqq \frac{(K-1)^{2}}{2} \sum_{j=0}^{n_{1}-1}\left(n_{1}-j\right) K^{j}=\frac{(K-1)^{2}}{2} \sum_{j=0}^{n_{1}-1}\left(\sum_{i=0}^{j} K^{i}\right) \\
& =\frac{(K-1)^{2}}{2} \sum_{j=0}^{n_{1}-1} \frac{K^{j+1}-1}{K-1}=\frac{K-1}{2}\left(\sum_{j=0}^{n_{1}-1} K^{j+1}-n_{1}\right) \\
& <\frac{K-1}{2}\left(\frac{K^{n_{1}}-1}{K-1} K\right)=\frac{K}{2}\left(K^{n_{1}}-1\right)<\frac{K}{2} x \text {, i.e., } t_{2}=O(x) \text {; } \\
& t_{3}=\frac{1}{2} \sum_{i=1}^{t} a_{i}\left(a_{i}-1\right) K^{n_{i}}<\frac{K-1}{2} \sum_{i=1}^{t} a_{i} K^{n_{i}}=\frac{K-1}{2} x \\
& \text { i.e., } t_{3}=O(x) \text {; }
\end{aligned}
$$




$$
\begin{aligned}
& t_{4}=\sum_{i=1}^{t} a_{i}<x, \text { i.e., } t_{4}=O(x) \\
& t_{5}=\sum_{i=2}^{t}\left(\sum_{j=1}^{i-1} a_{j}\right) a_{i} K^{n_{i}} \leqq(K-1)^{2} \sum_{i=2}^{t}(i-1) K^{n_{i}} \\
&=(K-1)^{2}\left(K^{n_{2}}+2 K^{n_{3}}+\cdots+(t-1) K^{n_{t}}\right) \\
& \leqq(K-1)^{2}\left[n_{1}+\left(n_{1}-1\right) K+\cdots+2 K^{n_{1}-2}+K^{n_{1}-1}\right] \\
&=(K-1)^{2}\left[\sum_{j=0}^{n_{1}-1}\left(\sum_{i=0}^{j} K^{i}\right)\right]=(K-1)^{2} \sum_{j=0}^{n-1} \frac{K^{j+1}-1}{K-1} \\
&=(K-1)\left(\sum_{j=0}^{n_{1}-1} K^{j+1}-n_{1}\right) \leqq K(K-1) \frac{K^{n_{1}}-1}{K-1} \leqq K x, \\
& K-\text { i.e, } t_{5}=O(x) .
\end{aligned}
$$

Therefore

$$
A(x)=\frac{K-1}{2} \frac{x \log x}{\log K}+O(x) .
$$

At the special case of $x=(K-1) K$,

$$
\frac{x \log x}{\log K}=\frac{(K-1) K^{n} n \log K-(K-1)}{\log K} .
$$

Hence

$$
\begin{aligned}
& \left(\frac{K-1}{2} \frac{x \log x}{\log K}-A(x)\right) / x=\left(\frac{(K-1)^{2}}{2} \frac{K^{n}[n \log K+\log (K-1)]}{\log K}\right. \\
& \left.-\frac{n(K-1)^{2} K^{n}}{2}-\frac{(K-1)(K-2) K^{n}}{2}-(K-1)\right) /(K-1) K^{n} \\
& =\frac{K-1}{2} \frac{\log (K-1)}{\log K}-\frac{K-2}{2}-\frac{1}{K^{n}} .
\end{aligned}
$$

As $n \rightarrow \infty$, the above formula's limit is larger than 0 . Hence we have

$$
\begin{aligned}
\limsup _{y \rightarrow \infty}\left(\frac{K-1}{2}\right. & \frac{\left.y \log \frac{y}{K}\right) / y}{\log K} \\
& \geqq \lim _{x=(K-1) K^{n}, n \rightarrow \infty}\left[\frac{K-1}{2} \frac{x \log x}{\log K}-A(x)\right] / x>0 .
\end{aligned}
$$

At the same time in the special case of $x=K^{n}$, it is easy to prove 


$$
\lim _{x \rightarrow \infty} A(x)-\frac{K-1}{2} \frac{x \log x}{\log K} / x=0 .
$$

This implies that our theorem is the best possible.

3. Proof of Theorem 2. Let $m$ be a fixed integer, then the equation $\alpha(y)=m$ has infinite solutions. Letting $B_{m}(x)$ be the number of solutions not larger than $x$, we have Theorem 2 .

THEOREM 2.

$$
B_{m}(x) \sim \frac{1}{m !}\left(\frac{\log x}{\log K}\right)^{m} .
$$

Proof. First we set $m \leqq K-1$ and $x=K^{n}$. If $y=a_{0}+a_{1} K+\cdots$ $+a_{n-1} K^{n-1}$ and $\alpha(y)=m$, then in the expansion of $\left(x_{0}+x_{1}+x_{2}+\cdots\right.$ $\left.+x_{n-1}\right)^{m}$, there must exist a term which is $x_{0}^{a_{0}} \cdots x_{n-1}^{a_{n-1}}$, or vice versa. This event shows that each term of the above formula's expansion has a one-to-one correspondence to $y$. Here $y$ can satisfy such relations: $\alpha(y)=m, y \leqq x$. But the above expansion has

$$
\left(\begin{array}{c}
n+m-1 \\
m
\end{array}\right)
$$

terms, and

$$
\left(\begin{array}{c}
n+m-1 \\
m
\end{array}\right) \sim \frac{n^{m}}{m !}=\frac{1}{m !}\left(\frac{\log x}{\log K}\right)^{m}, \quad(n \rightarrow \infty)
$$

so that our theorem is true with respect to this setting.

Secondly we set $m \leqq K-1$ and $K^{n-1}<x<K^{n}$.

$$
\begin{aligned}
\left(\frac{n-1}{n}\right)^{m} \frac{B\left(K^{n-1}\right)}{\frac{1}{m !}(n-1)^{m}} & =\frac{B\left(K^{n-1}\right)}{\frac{1}{m !} n^{m}}<\frac{B_{m}(x)}{\frac{1}{m !}\left(\frac{\log x}{\log K}\right)^{m}}<\frac{B_{m}\left(K^{n}\right)}{\frac{1}{m !}(n-1)^{m}} \\
& =\frac{B_{m}\left(K^{n}\right)}{\frac{n^{m}}{m}}\left(\frac{n}{n-1}\right)^{m} .
\end{aligned}
$$

The above inequality's both ends approach 1 as $n \rightarrow \infty$. From this result we can derive

$$
B_{m}(x) \sim \frac{1}{m !}\left(\frac{\log x}{\log K}\right)^{m}, \quad x \rightarrow \infty .
$$

Now we investigate the case of $m \geqq K$. With the same procedure 
we set $x=K^{n}$. We classify the expansion of $\left(x_{0}+x_{1}+\cdots+x_{n-1}\right)^{m}$ into $A$ and $B$. $A$ consists of such term $x_{0}^{a_{0}} x_{1}^{a_{1}} \cdots a_{n-1}^{a_{n-1}}$ which satisfies $a_{i} \leqq K-1(i=0, \cdots, n-1) ; B$ consists of the other terms which do not belong to $A$. It is readily seen that $B$ 's terms cannot be larger than $n$ times the terms of expansion of the following formula:

$$
\left(x_{0}+x_{1}+\cdots+x_{n-1}\right)^{m-K} .
$$

Hence $B$ 's terms are not larger than

$$
n \cdot\left(\begin{array}{c}
n+m-K-1 \\
m-K
\end{array}\right) .
$$

That is lower infinite of $n^{m} / m !$. In the beginning we speak of $K \geqq 2$.

It is easy to see that $y$, which can satisfy the relationship $\alpha(y)=m$, $K^{n-1}<x<K^{n}$, is one-to-one corresponding to every term of $B$. Therefore,

$$
B_{n}\left(K^{n}\right) \sim \frac{n^{m}}{m !}, \quad n \rightarrow \infty .
$$

It is the same as the second situation, and we have

$$
B_{m}(x) \sim \frac{1}{m !}\left(\frac{\log x}{\log K}\right)^{m},
$$

as $m \geqq K, K^{n-1}<x<K^{n}$. Our theorem is fully proved.

REFERENCE

1. R. Bellman and H. Shapiro, On a problem in additive number theory, Ann. of Math. (2) 49 (1948), 333-340.

Berkeley, California 